\title{
On the equivalence of mixed and behavior strategies in finitely additive decision problems
}

Citation for published version (APA):

Flesch, J., Vermeulen, D., \& Zseleva, A. (2019). On the equivalence of mixed and behavior strategies in finitely additive decision problems. Journal of Applied Probability, 56(3), 810-829.

https://doi.org/10.1017/jpr.2019.47

Document status and date:

Published: 01/09/2019

DOI:

10.1017/jpr.2019.47

Document Version:

Publisher's PDF, also known as Version of record

Document license:

Taverne

Please check the document version of this publication:

- A submitted manuscript is the version of the article upon submission and before peer-review. There can be important differences between the submitted version and the official published version of record.

People interested in the research are advised to contact the author for the final version of the publication, or visit the DOI to the publisher's website.

- The final author version and the galley proof are versions of the publication after peer review.

- The final published version features the final layout of the paper including the volume, issue and page numbers.

Link to publication

\footnotetext{
General rights rights.

- You may freely distribute the URL identifying the publication in the public portal. please follow below link for the End User Agreement:

www.umlib.nl/taverne-license

Take down policy

If you believe that this document breaches copyright please contact us at:

repository@maastrichtuniversity.nl

providing details and we will investigate your claim.
}

Copyright and moral rights for the publications made accessible in the public portal are retained by the authors and/or other copyright owners and it is a condition of accessing publications that users recognise and abide by the legal requirements associated with these

- Users may download and print one copy of any publication from the public portal for the purpose of private study or research.

- You may not further distribute the material or use it for any profit-making activity or commercial gain

If the publication is distributed under the terms of Article $25 \mathrm{fa}$ of the Dutch Copyright Act, indicated by the "Taverne" license above, 


\title{
ON THE EQUIVALENCE OF MIXED AND BEHAVIOR STRATEGIES IN FINITELY ADDITIVE DECISION PROBLEMS
}

\author{
JÁNOS FLESCH, ${ }^{* * *}$ \\ DRIES VERMEULEN, ${ }^{*} * * *$ Maastricht University AND \\ ANNA ZSELEVA, ${ }^{* * * * * * * * *}$ Tel Aviv University
}

\begin{abstract}
We consider decision problems with arbitrary action spaces, deterministic transitions, and infinite time horizon. In the usual setup when probability measures are countably additive, a general version of Kuhn's theorem implies under fairly general conditions that for every mixed strategy of the decision maker there exists an equivalent behavior strategy. We examine to what extent this remains valid when probability measures are only assumed to be finitely additive. Under the classical approach of Dubins and Savage (2014), we prove the following statements: (1) If the action space is finite, every mixed strategy has an equivalent behavior strategy. (2) Even if the action space is infinite, at least one optimal mixed strategy has an equivalent behavior strategy. The approach by Dubins and Savage turns out to be essentially maximal: these two statements are no longer valid if we take any extension of their approach that considers all singleton plays.

Keywords: Mixed strategy; behavior strategy; finitely additive probability measure; equivalent strategy; Kuhn's theorem
\end{abstract}

2010 Mathematics Subject Classification: Primary 91B06

Secondary $91 \mathrm{~A} 18$

\section{Introduction}

We consider dynamic decision problems in which the decision maker has to choose actions sequentially, at infinitely many periods. At each period, the decision maker can make their choice of action depend on the history consisting of all previously chosen actions. A strategy is a decision rule that gives a recommendation to the decision maker on how to choose actions during the decision problem. These recommendations, however, can be given in essentially different ways, both from a conceptual and from a technical point of view. As usual, we distinguish three main types of strategies: pure strategies, mixed strategies, and behavior strategies.

\footnotetext{
Received 6 November 2018; revision received 13 May 2019.

* Postal address: School of Business and Economics, Department of Quantitative Economics, Maastricht University, PO Box 616, 6200 MD Maastricht, The Netherlands.

** Email address: j.flesch@maastrichtuniversity.nl

*** Email address: d.vermeulen@maastrichtuniversity.nl

**** Postal address: School of Mathematical Sciences, Tel Aviv University, 6997800 Tel Aviv, Israel.

***** Email address: zseleva.anna@gmail.com

Support from the Basic Research Program of the National Research University Higher School of Economics is gratefully acknowledged.
} 
A pure strategy recommends one action at every decision point, depending on the history. This recommendation is fully deterministic.

A mixed strategy is a probability measure on the set of pure strategies. The interpretation is that the decision maker should first draw a pure strategy according to this probability measure, and then play the decision problem by following the recommendations of this pure strategy. Thus, a mixed strategy uses randomization, but only once and at the very beginning of the decision problem.

A behavior strategy specifies a probability measure on the set of actions, depending on the history. The interpretation is that, at the current decision point, the decision maker should choose their action according to this probability measure. Thus, a behavior strategy uses randomization at every decision point, and consequently involves successive usage of probability measures.

In the usual setup where probability measures are countably additive, mixed strategies and behavior strategies are strongly connected, under fairly general conditions. Both mixed strategies and behavior strategies induce - albeit in a different way - a probability measure on the set of all possible sequences of actions, called plays. A mixed strategy is equivalent to a behavior strategy if the induced probability measures are identical. This of course implies that the induced expected payoffs are also equal, up to a measurability condition on the payoff function. In the countably additive setup a fundamental theorem, a general version of Kuhn's theorem by Aumann (1964), implies under fairly general conditions that for every mixed strategy there exists an equivalent behavior strategy. This allows us, in most models, to simply focus on behavior strategies.

A less common, though well-established, alternative is to only assume that probability measures are finitely additive. Weakening the requirement of countable additivity to finite additivity of course has its consequences - see the overview given later in this section. One notable difference is that the strategic possibilities of the decision maker become richer: generally, both the set of mixed strategies and the set of behavior strategies are strictly larger in the finitely additive setup. This leads naturally to the question whether, or under what conditions, finitely additive mixed and behavior strategies are equivalent. Our goal is to address this question.

In this paper we only assume that probability measures are finitely additive. From now on, we refer to finitely additive probability measures as charges. In this setup, a mixed strategy is a charge on the set of pure strategies, and a behavior strategy is a mapping that assigns to each history a charge on the action space.

Since each pure strategy induces a unique play, a mixed strategy naturally induces a charge on the set of plays. For a behavior strategy, however, it is not immediately obvious how to define the induced charge on the set of plays. Various alternatives have been considered in the literature, motivated mainly by conceptual but to some extent also technical reasons; see, for example, Dubins and Savage (2014), Dubins (1974), and Purves and Sudderth (1976). A classical approach to this question is given by Dubins and Savage (2014), whose focus is on the the algebra of all clopen (closed and open) sets of plays, when the set of actions is endowed with the discrete topology and the set of plays is endowed with the product topology. Then, for each behavior strategy they define a natural charge on this algebra. This approach is rather minimal in the sense that essentially all later papers in the literature agree to define the induced charge on this algebra exactly the same way, and only consider the question of how to extend it to larger algebras.

The general approach to define the charge induced by a behavior strategy on the set of plays goes as follows: First, fix an algebra on the set of plays. Second, given this algebra, specify for each behavior strategy a certain charge on this algebra. 
The choice of the algebra and the specification of a charge for each behavior strategy is important, and as we will see, the results can depend on them. So one may therefore wonder how to choose them. The specific choice of the algebra can depend on conceptual grounds, e.g. which sets we find natural and which sets we think should be assigned a probability under each behavior strategy. But this choice can also be motivated from a technical point of view, e.g. what type of sets we want to consider, and later what type of charges we want to define for behavior strategies. The choice of the specification of a charge on this algebra for each behavior strategy is again both a conceptual and a technical matter.

Given an algebra on the set of plays and a specification of a charge on this algebra for each behavior strategy, we define equivalence between mixed and behavior strategies. We say that a mixed strategy and a behavior strategy are equivalent whenever they generate the same charge on the chosen algebra.

Moreover, if the payoff function is integrable with respect to this algebra, then each behavior strategy induces a unique expected payoff. This allows us to define optimality for behavior strategies.

\subsection{Overview of our contribution}

In this paper we show the following results on the equivalence between mixed and behavior strategies.

(i) Every behavior strategy has an equivalent mixed strategy, under general conditions (see Theorem 2).

(ii) If the action space is finite, every mixed strategy has an equivalent behavior strategy under the classical approach given by Dubins and Savage (2014) on the algebra of all clopen sets (see Theorem 4). This algebra turns out to be essentially maximal: for any larger algebra that contains each singleton play, there is a mixed strategy that has no equivalent behavior strategy (see Theorem 6). This negative result also applies to the Borel sigma-algebra, which plays an important role in various models.

We remark that if the action space is infinite, it follows from an example in Dubins (1975) that there is a mixed strategy without an equivalent behavior strategy (see Theorem 3), even under the approach of Dubins and Savage (2014).

In addition, we also investigate how far the equivalence between mixed and behavior strategies goes when we only consider optimal mixed and optimal behavior strategies. It is of course always true that if a mixed strategy is optimal and has an equivalent behavior strategy, then this behavior strategy is also optimal. Conversely, if a behavior strategy is optimal, then every equivalent mixed strategy is also optimal. We show the following results:

(iii) An optimal mixed strategy always exists (see Theorem 1). (An optimal pure strategy does not necessarily exist. Indeed, whenever the payoff function has no maximum, the decision maker has no optimal pure strategy.)

(iv) An optimal behavior strategy always exists under the approach by Dubins and Savage (2014), even if the action space is infinite (see Theorem 5). Thus, there always exists an optimal mixed strategy that has an equivalent behavior strategy under the approach by Dubins and Savage.

(v) The clopen algebra considered in Dubins and Savage (2014) turns out to be essentially maximal: for any larger algebra that contains each singleton play there is a payoff 
function for which the decision maker has no optimal behavior strategy (see Theorem 8). That is, behavior strategies strictly underperform compared to mixed strategies.

To sum up, every behavior strategy has an equivalent mixed strategy, but not necessarily the other way around. There is always an optimal mixed strategy; however, an optimal behavior strategy does not always exist. Generally speaking, our results suggest that mixed strategies may be more suitable for studying finitely additive decision problems.

\subsection{Related literature}

1.2.1. Finite additivity. There are different ways of defining probabilities. Countable additivity is a usual assumption of probability. However, from a conceptual point of view, the weaker assumption of finite additivity has also been argued for; see, for example, Savage (1972), de Finetti (1975), and Dubins and Savage (2014). Since finite additivity is the weaker assumption, it is also more basic. It allows for a richer class of probabilities, and facilitates constructions such as a uniform probability distribution over the natural numbers (see Schirokauer and Kadane (2007)). For a more extensive summary of the history of finite additivity and its relation to countable additivity, see Bingham (2010).

1.2.2. Dynamic decision problems. As mentioned earlier, Dubins and Savage (2014) studied the same type of decision problems as we do, and more concretely the algebra generated by the clopen sets. Dubins (1974) considered the same type of problems, but considered the algebra generated by the open sets. Purves and Sudderth (1976) went further, considering the Borel sigma-algebra.

Sudderth (2016) wrote about finitely additive dynamic programming. His model includes finitely additive strategies and an infinite time horizon, as does ours. However, his model has states and probabilistic transitions, and the payoff is some type of aggregation of daily payoffs, for example by discounting. The paper also contains comparisons with the countably additive theory. For example, it shows that there are cases when there exists a finitely additive optimal stationary strategy; in contrast, a countably additive one need not exist.

Charges also gained recognition in game theory, such as in Maitra and Sudderth (1993), Marinacci (1997), Maitra and Sudderth (1998), Harris et al. (2005), Capraro and Scarsini (2013), and Flesch et al. (2017), but to a lesser extent.

1.2.3. Equivalent strategies. Equivalence between mixed and behavior strategies was originally investigated by Kuhn (1953), and in a more general version by Aumann (1964). We also refer to Takahashi (1969) and Maschler et al. (2013), Theorem 6.15. Muraviev et al. (2017) extended Kuhn's result to Ellsberg decision problems. Aryal and Stauber (2014) showed the limits of extending Kuhn's theorem in the case of ambiguity-averse players. In the finitely additive setup, as mentioned above, Dubins (1975) showed a mixed strategy without an equivalent behavior strategy in a decision problem with an infinite action space.

\subsection{Structure of the paper}

In Section 2 we discuss some preliminaries on charges. In Section 3 we present the model. In Section 4 we define the charges that mixed and respectively behavior strategies induce on the set of plays, which leads to the definition of equivalence between mixed and behavior strategies, as well as to expected payoffs and the definition of optimality. Our investigation of the equivalence between mixed and behavior strategies is in Section 5 under the approach of Dubins and Savage, and in Section 6 on algebras that are larger than the clopen algebra they considered. 


\section{Preliminaries}

In this section we provide a brief summary of (probability) charges. For further reading, we refer to Dunford and Schwartz (1964) and Rao and Rao (1983).

\subsection{Charges}

Let $X$ be a nonempty set and let $\mathcal{P}$ be an algebra on $X$. A probability charge, or simply charge, on $(X, \mathcal{P})$ is a function $\mu: \mathcal{P} \rightarrow[0,1]$ such that $\mu(X)=1$ and, for all disjoint sets $E, F \in \mathcal{P}, \mu(E \cup F)=\mu(E)+\mu(F)$. A charge is thus finitely additive, but not necessarily countably additive. We denote the set of all charges on $(X, \mathcal{P})$ by $\mathcal{C}(X, \mathcal{P})$, and use the notation $\mu(x)$ instead of $\mu(\{x\})$.

When $X$ is countably infinite, we say that $\mu \in \mathcal{C}\left(X, 2^{X}\right)$ is a diffuse charge if $\mu(x)=0$ for every $x \in X$. Diffuse charges are not countably additive. It follows from the axiom of choice that diffuse charges exist (see, for example, Rao and Rao (1983), p. 38).

The following statement follows from Theorem 2 in Los and Marczewski (1949) together with the axiom of choice: If $\mathcal{P}$ is an algebra on $X$ and $\mu$ is a charge on $(X, \mathcal{P})$, then there exists a charge $v$ on $\left(X, 2^{X}\right)$ such that $\nu(E)=\mu(E)$ for all $E \in \mathcal{P}$. We say that $\nu$ extends $\mu$.

\subsection{Integration with respect to charges}

Let $\mathcal{P}$ be an algebra on $X$. A function $s: X \rightarrow \mathbb{R}$ is called a $\mathcal{P}$-measurable simple function if $s=\sum_{i=1}^{m} c_{i} \mathbb{I}_{B_{i}}$, where $c_{1}, \ldots, c_{m} \in \mathbb{R},\left\{B_{1}, \ldots, B_{m}\right\}$ is a $\mathcal{P}$-measurable partition of $X$, and $\mathbb{I}_{B_{i}}$ is the characteristic function of the set $B_{i}$. Let $\mu$ be a charge on $(X, \mathcal{P})$. The integral of $s$ with respect to $\mu$ is defined by $\int_{X} s \mathrm{~d} \mu=\sum_{i=1}^{m} c_{i} \cdot \mu\left(B_{i}\right)$.

Let $\mu$ be a charge on $(X, \mathcal{P})$. For a bounded function $f: X \rightarrow \mathbb{R}$, the lower integral of $f$ with respect to $\mu$ is defined as the supremum of all real numbers $\int_{X} s \mathrm{~d} \mu$, where $s$ is a $\mathcal{P}$ measurable simple function with $s \leq f$. The upper integral of $f$ is defined analogously. The function $f: X \rightarrow \mathbb{R}$ is called $\mathcal{P}$-integrable if for each $\mu \in \mathcal{C}(X, \mathcal{P})$, the lower and the upper integrals of $f$ with respect to $\mu$ coincide. It follows from Theorems 4.5.7 and 4.7.3 in Rao and Rao (1983) that $f$ is $\mathcal{P}$-integrable if and only if for every $\varepsilon>0$ there exists a $\mathcal{P}$-measurable simple function $f^{\prime}: P \rightarrow \mathbb{R}$ such that $\left|f(p)-f^{\prime}(p)\right| \leq \varepsilon$ for every $p \in P$. Consequently, any bounded function $f: X \rightarrow \mathbb{R}$ is $2^{X}$-integrable. $\mathcal{P}$-integrability is sometimes called uniform $\mathcal{P}$ approachability of $f$. Similar conditions also appear for example in Section 4 of Marinacci (1997) and Section 2.1.3 of Harris et al. (2005), albeit in a different context. Notice that if the function $f$ is $\mathcal{P}$-integrable, then it is also $\mathcal{P}^{\prime}$-integrable for all $\mathcal{P}^{\prime} \supset \mathcal{P}$.

\section{The model}

\subsection{The decision problem}

Let $A$ be an action set having at least two elements. Let $H$ denote the set of finite sequences in $A$, including the empty sequence $\emptyset$. Let $P$ denote the set of infinite sequences in $A$. Elements of $A$ are called actions, elements of $H$ are called histories, and elements of $P$ are called plays. Let $u: P \rightarrow \mathbb{R}$ be a bounded function, called the payoff function.

Consider the following decision problem. At each period $t=1,2, \ldots$, the decision maker chooses an action $a_{t}$ from $A$, knowing the previous choices $\left(a_{1}, \ldots, a_{t-1}\right) \in H$. This induces a play $p=\left(a_{1}, a_{2}, \ldots\right)$. The payoff of the decision maker is $u(p)$. (Note that the set of available actions does not depend on the history of the decision problem, and is always $A$. Moreover, notice that our model is equivalent to a model with a state variable and deterministic transitions.) 
A strategy is a decision rule that gives a recommendation to the decision maker for choosing actions. These recommendations can be given in essentially different ways, and based on that we distinguish three different types of strategies: pure strategies, mixed strategies, and behavior strategies. We discuss each type of strategy in turn.

\subsection{Pure strategies}

A pure strategy is a function $f: H \rightarrow A$. The interpretation is that if history $h$ arises during the decision problem, then the strategy $f$ recommends that the decision maker choose action $f(h)$. Note that a pure strategy is completely deterministic.

Each pure strategy $f$ induces a unique play $p_{f}$. Of course, two different pure strategies can induce the same play, but any play can be generated by at least one pure strategy. We denote the set of pure strategies by $F$.

\subsection{Mixed strategies}

A mixed strategy is a charge $m \in \mathcal{C}\left(F, 2^{F}\right)$ on the set of pure strategies. The interpretation is that the decision maker should draw a pure strategy $f$ according to $m$, and play the decision problem by using $f$. Note that a pure strategy can be seen as a mixed strategy that places probability 1 on this pure strategy.

\subsection{Behavior strategies}

A behavior strategy is a function $b: H \rightarrow \mathcal{C}\left(A, 2^{A}\right)$. The interpretation is that if history $h$ arises during the decision problem, then the strategy $b$ recommends that the decision maker choose an action according to the charge $b(h)$.

Thus, a behavior strategy successively uses an infinite sequence of charges during the play. Consequently, a behavior strategy that places probability 1 on an action $a_{t}$ at every period $t$ does not necessarily induce the play $\left(a_{1}, a_{2}, \ldots\right)$ with probability 1 in our finitely additive setting. So, a pure strategy cannot necessarily be identified with the behavior strategy placing probability 1 on the corresponding actions. This is in sharp contrast with the countably additive setup (see Section 6).

\section{Induced charges on the set $P$ of plays, expected payoffs, and optimality}

In this section we define and discuss the charges that mixed and respectively behavior strategies induce on the set $P$ of plays. This allows us to calculate expected payoffs, define optimality, and define equivalence between mixed and behavior strategies.

\subsection{Mixed strategies}

In this subsection we consider mixed strategies. First, we define the charge that a mixed strategy induces on a set of plays. This allows us to calculate the expected payoff for each mixed strategy and define optimal mixed strategies. Finally, we show that an optimal mixed strategy always exists.

A pure strategy $f$ induces a unique play $p_{f}$. Naturally, a mixed strategy $m$ generates a charge $m^{*}$ on $\left(P, 2^{P}\right)$, given for every $Q \subseteq P$ by

$$
m^{*}(Q)=m\left(\left\{f \in F: p_{f} \in Q\right\}\right) .
$$

Note that, as different pure strategies can induce the same play, different mixed strategies can induce the same charge on $\left(P, 2^{P}\right)$. 
A pure strategy $f$ induces the payoff $U(f)=u\left(p_{f}\right)$. As a mixed strategy $m$ prescribes drawing a pure strategy $f$ according to the charge $m$ defined on $\left(F, 2^{F}\right)$, the expected payoff for the mixed strategy $m$ is given by

$$
U(m)=\int_{f \in F} U(f) m(\mathrm{~d} f) .
$$

The next lemma claims that the expected payoff for a mixed strategy can also be calculated through the induced charge on $\left(P, 2^{P}\right)$. The proof is straightforward and therefore omitted.

Lemma 1. For every mixed strategy $m$,

$$
U(m)=\int_{p \in P} u(p) m^{*}(\mathrm{~d} p) .
$$

We call a pure strategy $f$ optimal if $U(f)=\sup _{p \in P} u(p)$. Similarly, we call a mixed strategy $m$ optimal if $U(m)=\sup _{p \in P} u(p)$.

It is not always true that there exists a pure optimal strategy, since the payoff function $u$ might not have a maximum, for example when the range of $u$ is the set $\{1-1 / n: n \in \mathbb{N}\}$. However, it is easy to see that an optimal mixed strategy always exists.

Theorem 1. An optimal mixed strategy always exists.

Proof. Let $U^{*}=\sup _{p \in P} u(p)$. For each $n \in \mathbb{N}$, let $p_{n} \in P$ be a play such that $u\left(p_{n}\right) \geq U^{*}-$ $\frac{1}{n}$, and let $f_{n}$ denote a pure strategy that induces the play $p_{n}$.

Let $\tau$ be a diffuse charge on $\left(\mathbb{N}, 2^{\mathbb{N}}\right)$. Now consider the mixed strategy $m$ that, intuitively, first chooses a natural number $n \in \mathbb{N}$ according to $\tau$ and then plays the pure strategy $f_{n}$. Formally, for every subset $F^{\prime}$ of the set $F$ of pure strategies we have

$$
m\left(F^{\prime}\right)=\tau\left(\left\{n \in \mathbb{N} \mid f_{n} \in F^{\prime}\right\}\right)
$$

Let $K \in \mathbb{N}$. Since

$$
m\left(\left\{f_{K}, f_{K+1}, \ldots\right\}\right)=\tau(\{K, K+1, \ldots\})=1,
$$

it follows from (2) that

$$
U(m)=\int_{f \in F} U(f) m(\mathrm{~d} f)=\int_{f \in\left\{f_{K}, f_{K+1}, \ldots\right\}} U(f) m(\mathrm{~d} f) \geq U^{*}-\frac{1}{K} .
$$

As this holds for every $K \in \mathbb{N}$, we have $U(m)=U^{*}$.

\subsection{Behavior strategies}

In this subsection we turn to behavior strategies. We consider algebras on the set of plays that satisfy a minimal richness condition. We call such algebras competent. Then, we define the charge that a behavior strategy induces on such a competent algebra. Based on these induced charges, we define optimal behavior strategies.

For a history $h \in H$ and a play $p \in P$ we use the notation $h \prec p$ if the play $p$ starts with the history $h$. For two histories $h, h^{\prime} \in H$ we use the notation $h \preceq h^{\prime}$ if the history $h^{\prime}$ starts with $h$ (allowing $h^{\prime}=h$ ), and use the notation $h \prec h^{\prime}$ if $h^{\prime}$ starts with $h$ and $h^{\prime}$ is strictly longer than $h$. 
We now discuss the algebras that we consider on the set of plays. Arguably, a minimal requirement on the algebra is that it should contain all subsets of plays that are determined in finite time. More precisely, for every $n \in \mathbb{N}$ and $S \subseteq A^{n}$, the algebra should contain the set

$$
[S]=\{p \in P \mid \text { there exists } h \in S \text { with } h \prec p\} .
$$

Thus, a play $p=\left(a_{1}, a_{2}, \ldots\right)$ belongs to $[S]$, where $S \subseteq A^{n}$, if and only if $\left(a_{1}, \ldots, a_{n}\right)$ belongs to $S$. For the special case when $S=\left\{\left(a_{1}, a_{2}, \ldots, a_{n}\right)\right\}$ is a singleton set, for convenience we write $\left[a_{1}, a_{2}, \ldots, a_{n}\right]$ instead of $\left[\left\{\left(a_{1}, a_{2}, \ldots, a_{n}\right)\right\}\right]$.

Let $\mathcal{R}^{\mathrm{f}}$ denote the set of all sets $[S]$, where $S \subseteq A^{n}$ for some $n \in \mathbb{N}$. The superscript f refers to a finite horizon. The set $\mathcal{R}^{\mathrm{f}}$ consists of the so-called 'finite-dimensional' subsets of the set $P$ of plays. The following statement is well known and easy to verify.

Lemma 2. $\mathcal{R}^{\mathrm{f}}$ is an algebra.

Take a behavior strategy $b$ and a set $[S]$, where $S \subseteq A^{n}$ for some $n \in \mathbb{N}$. It is natural and standard to calculate the probability of the set $[S]$ under $b$ through $n$ iterated integrals as follows: let

$$
c^{\mathrm{f}}(b)([S])=\int_{a_{1} \in A} \cdots \int_{a_{n} \in A} \mathbb{I}_{S}\left(a_{1}, \ldots, a_{n}\right) b\left(a_{1}, \ldots, a_{n-1}\right)\left(\mathrm{d} a_{n}\right) \cdots b(\varnothing)\left(\mathrm{d} a_{1}\right) .
$$

Note that $c^{\mathrm{f}}(b)$ is a charge on the algebra $\mathcal{R}^{\mathrm{f}}$. In the language of Dubins and Savage, $c^{\mathrm{f}}(b)([S])$ is nothing but the strategic probability of $[S]$.

Definition 1. An algebra $\mathcal{P}$ of $P$ is called competent if $\mathcal{P} \supseteq \mathcal{R}^{\mathrm{f}}$. Given a competent algebra $\mathcal{P}$, a $\mathcal{P}$-specification is a function $\psi^{\mathcal{P}}$ that to each behavior strategy $b$ assigns a charge $\psi^{\mathcal{P}}(b)$ on $(P, \mathcal{P})$ with the requirement that, for all sets $[S] \in \mathcal{R}^{\mathrm{f}}$,

$$
\psi^{\mathcal{P}}(b)([S])=c^{\mathrm{f}}(b)([S]) .
$$

Thus, given a competent algebra $\mathcal{P}$ and a behavior strategy $b$, a $\mathcal{P}$-specification $\psi^{\mathcal{P}}$ assigns a probability $\psi^{\mathcal{P}}(b)(R)$ to each set $R$ in the algebra $\mathcal{P}$, the only requirement being that $\psi^{\mathcal{P}}$ coincides with $c^{\mathrm{f}}$ on the algebra $\mathcal{R}^{\mathrm{f}}$.

Note that $\mathcal{R}^{\mathrm{f}}$, in view of Lemma 2 , is a competent algebra of $P$, and $c^{\mathrm{f}}$, defined in (3), is an $\mathcal{R}^{\mathrm{f}}$-specification.

Let $\mathcal{P}$ be a competent algebra of $P$, and let $\psi^{\mathcal{P}}$ be a $\mathcal{P}$-specification. Assume that the payoff function $u$ is $\mathcal{P}$-integrable. Then, for every behavior strategy $b$, the payoff function $u$ has the same lower and upper integrals with respect to $\psi^{\mathcal{P}}(b)$, which we denote by $U\left(b \mid \mathcal{P}, \psi^{\mathcal{P}}\right)$. Based on this observation, we can define optimality for behavior strategies.

Definition 2. Let $\mathcal{P}$ be a competent algebra of $P$, and let $\psi^{\mathcal{P}}$ be a $\mathcal{P}$-specification. Assume that the payoff function $u$ is $\mathcal{P}$-integrable. A behavior strategy $b$ is called $\left(\mathcal{P}, \psi^{\mathcal{P}}\right)$-optimal if $U\left(b \mid \mathcal{P}, \psi^{\mathcal{P}}\right)=\sup _{p \in P} u(p)$.

\subsection{Equivalence of strategies}

Consider a competent algebra $\mathcal{P}$ on $P$. Each mixed strategy $m$ induces a charge $m^{\mathcal{P}}$ on $(P, \mathcal{P})$, which is the restriction of the charge $m^{*}$ defined in (1) to the algebra $\mathcal{P}$. Also, given a $\mathcal{P}$-specification $\psi^{\mathcal{P}}$, each behavior strategy $b$ induces a charge $\psi^{\mathcal{P}}(b)$ on $(P, \mathcal{P})$. This allows us to define equivalence between strategies. 
Definition 3. Let $\mathcal{P}$ be a competent algebra of $P$, and let $\psi^{\mathcal{P}}$ be a $\mathcal{P}$-specification. A mixed strategy $m$ and a behavior strategy $b$ are said to be $\left(\mathcal{P}, \psi^{\mathcal{P}}\right)$-equivalent if they generate the same charge on $(P, \mathcal{P})$, i.e. $m^{\mathcal{P}}(Q)=\psi^{\mathcal{P}}(b)(Q)$ for every set $Q \in \mathcal{P}$.

According to the next theorem, we can find an equivalent mixed strategy for every behavior strategy under general conditions. The proof can be found in the Appendix.

Theorem 2. Let $\mathcal{P}$ be an algebra of $P$, and let $\psi^{\mathcal{P}}$ be a $\mathcal{P}$-specification. Then for every behavior strategy there exists a $\left(\mathcal{P}, \psi^{\mathcal{P}}\right)$-equivalent mixed strategy.

The converse, when for a given mixed strategy we try to find an equivalent behavior strategy, is much more complicated. This is highlighted by a two-period decision problem in Section 2 of Dubins (1975), with action space $\mathbb{N}$ at period 1 and only two actions at period 2 . In this decision problem there is a mixed strategy for which there is no $\left(\mathcal{R}^{\mathrm{f}}, c^{\mathrm{f}}\right)$-equivalent behavior strategy. This example is rather minimal, since for lack of equivalence at least two time periods are needed and in at least one time period there have to be infinitely many actions. For the proof Dubins refers to an implicit argument in de Finetti (1972). The following theorem follows directly from this example. Since our terminology is different, we provide a proof along the construction of Dubins (1975).

Theorem 3. Assume that the action space is infinite. Then there is a mixed strategy that has no $\left(\mathcal{P}, \psi^{\mathcal{P}}\right)$-equivalent behavior strategy for any competent algebra $\mathcal{P}$ and $\mathcal{P}$-specification $\psi^{\mathcal{P}}$.

Proof. For simplicity, assume that $\mathbb{N} \subseteq A$. For each $n \in \mathbb{N}$, consider pure strategies $f_{n}$ and $g_{n}$ that induce the plays $p_{n}=(n, 1,1, \ldots)$ and $q_{n}=(n, 2,2, \ldots)$, respectively. Let $\tau^{f}$ be a diffuse charge on $\left(\mathbb{N}, 2^{\mathbb{N}}\right)$ and let $\tau^{g}$ be the countably additive probability measure on $\left(\mathbb{N}, 2^{\mathbb{N}}\right)$ with $\tau^{g}(n)=\frac{1}{2^{n}}$ for each $n \in \mathbb{N}$.

Following Dubins (1975), we define a mixed strategy $m$ that, intuitively, gives a recommendation for a pure strategy in the following way: (1) with probability $\frac{1}{2}$, it chooses $n \in \mathbb{N}$ according to $\tau^{f}$ and recommends the pure strategy $f_{n}$; and (2) with probability $\frac{1}{2}$, it chooses $n \in \mathbb{N}$ according to $\tau^{g}$ and recommends the pure strategy $g_{n}$.

More precisely, for every subset $F^{\prime}$ of the set of pure strategies $F$ we have

$$
m\left(F^{\prime}\right)=\frac{1}{2} \cdot \tau^{f}\left(\left\{n \in \mathbb{N} \mid f_{n} \in F^{\prime}\right\}\right)+\frac{1}{2} \cdot \tau^{g}\left(\left\{n \in \mathbb{N} \mid g_{n} \in F^{\prime}\right\}\right) .
$$

This mixed strategy $m$ does not have a $\left(\mathcal{P}, \psi^{\mathcal{P}}\right)$-equivalent behavior strategy for any competent algebra $\mathcal{P}$ and $\mathcal{P}$-specification $\psi^{\mathcal{P}}$. Indeed, suppose by way of contradiction that a behavior strategy $b$ is $\left(\mathcal{P}, \psi^{\mathcal{P}}\right)$-equivalent to $m$ for some competent algebra $\mathcal{P}$ and $\mathcal{P}$-specification $\psi^{\mathcal{P}}$.

Step 1. For every $W \in \mathcal{P}$ it holds that

$$
m^{*}(W)=\frac{1}{2} \cdot \tau^{f}\left(\left\{n \in \mathbb{N} \mid p_{n} \in W\right\}\right)+\frac{1}{2} \cdot \tau^{g}\left(\left\{n \in \mathbb{N} \mid q_{n} \in W\right\}\right) .
$$

Consequently, we have the following under the mixed strategy $m$ for every $n \in \mathbb{N}$ :

[1] The first action is $n$ with probability

$$
m^{*}([n])=\frac{1}{2} \cdot \tau^{f}(n)+\frac{1}{2} \cdot \tau^{g}(n)=0+\frac{1}{2^{n+1}}=\frac{1}{2^{n+1}} .
$$


[2] The histories $(n, 1)$ and $(n, 2)$ have probability

$$
m^{*}([n, 1])=\frac{1}{2} \cdot \tau^{f}(n)=0 \quad \text { and } \quad m^{*}([n, 2])=\frac{1}{2} \cdot \tau^{g}(n)=\frac{1}{2^{n+1}} .
$$

Note that the sets $[n],[n, 1]$, and $[n, 2]$ belong to $\mathcal{P}$, as the algebra $\mathcal{P}$ is competent.

Step 2. Since the behavior strategy $b$ is $\left(\mathcal{P}, \psi^{\mathcal{P}}\right)$-equivalent to $m$, we have for every $n \in \mathbb{N}$ that

$$
b(\varnothing)(n)=\frac{1}{2^{n+1}}, \quad b(n)(1)=0, \quad b(n)(2)=1 .
$$

That is, $b$ recommends to choose action $n$ at period 1 with probability $\frac{1}{2^{n+1}}$, and after choosing any action $n$ at period $1, b$ recommends to choose action 2 at period 2 .

Step 3. Let $W$ be the set of plays that have action 2 at period 2. Since the algebra $\mathcal{P}$ is competent, $W \in \mathcal{P}$. Because $b(n)(2)=1$ for all $n \in \mathbb{N}$, we have $\psi^{\mathcal{P}}(b)(W)=1$, which, however, contradicts $m^{*}(W)=\frac{1}{2}$.

Thus, $m$ has no $\left(\mathcal{P}, \psi^{\mathcal{P}}\right)$-equivalent behavior strategy, as claimed above.

\section{The approach to behavior strategies by Dubins and Savage}

We defined equivalence between mixed and behavior strategies based on the choices of the competent algebra on the set of plays and the specification that assigns a probability to each set in the algebra under any behavior strategy. A classical approach was given by Dubins and Savage (2014), who proposed taking the algebra of all clopen (closed and open) sets of plays and a corresponding specification with a consistency property. (Dubins and Savage did not use the terminology 'specification'.) This section is devoted to this approach.

\subsection{The algebra $\mathcal{R}^{\mathrm{DS}}$ of clopen sets and the $\mathcal{R}^{\mathrm{DS}}$-specification $\psi^{\mathrm{DS}}$}

Following Dubins and Savage, we endow the set $A$ of actions with the discrete topology and the set $P$ of plays with the induced product topology. In this topology, a set $Q \subseteq P$ is open exactly when for every play $p \in Q$ there is a history $h \prec p$ such that $[h] \subseteq Q$. This topological space is completely metrizable, and it is compact if and only if $A$ is finite (see Kechris (1995)).

Let $\mathcal{R}^{\mathrm{DS}}$ be the set of all clopen subsets of $P$ (the superscript DS refers to Dubins and Savage). The following lemma summarizes the basic properties of $\mathcal{R}^{\mathrm{DS}}$ (see also Theorem 2.9.1 in Dubins and Savage (2014)). The proofs are straightforward and are therefore omitted.

Lemma 3. The collection $\mathcal{R}^{\mathrm{DS}}$ has the following properties: (1) $\mathcal{R}^{\mathrm{DS}}$ is an algebra. (2) $\mathcal{R}^{\mathrm{DS}} \supseteq$ $\mathcal{R}^{\mathrm{f}}$, and hence $\mathcal{R}^{\mathrm{DS}}$ is competent. (3) $\mathcal{R}^{\mathrm{DS}}=\mathcal{R}^{\mathrm{f}}$ if and only if the action set $\mathrm{A}$ is finite.

Now we turn to the specification suggested by Dubins and Savage. In order to state it precisely, we need some notation.

Consider a decision problem $G$ with action set $A$ and payoff function $u$. For a period $k \in \mathbb{N}$ and a history $h \in A^{k}$, we denote the subproblem that starts at history $h$ by $\left.G\right|_{h}$. The subproblem is played as follows. At periods $n \geq k+1$, the decision maker chooses an action $a_{n} \in A$, which induces a play $\left(a_{k+1}, a_{k+2}, \ldots\right)$ and a corresponding payoff $\left.u\right|_{h}\left(a_{k+1}, a_{k+2}, \ldots\right)=$ $u\left(h, a_{k+1}, a_{k+2}, \ldots\right)$. Thus, $\left.G\right|_{h}$ can be seen as the continuation of the decision problem $G$ given that the history $h$ has arisen. In fact, $\left.G\right|_{h}$ is a decision problem itself according to our 
definition (up to the inessential change that the first period has label $k+1$ ), with action set $A$ and payoff function $\left.u\right|_{h}$. Note that the set of plays is also $P=A^{\mathbb{N}}$ in $\left.G\right|_{h}$. It is easy to verify that if $Q$ is a clopen set of plays, then the continuation of $Q$ in the subproblem $\left.G\right|_{h}$,

$$
\left.Q\right|_{h}=\left\{\left(a_{k+1}, a_{k+2}, \ldots\right):\left(h, a_{k+1}, a_{k+2}, \ldots\right) \in Q\right\},
$$

is also clopen. That is, if $Q \in \mathcal{R}^{\mathrm{DS}}$ then $\left.Q\right|_{h} \in \mathcal{R}^{\mathrm{DS}}$.

A behavior strategy $b$ in the decision problem $G$ induces a behavior strategy $\left.b\right|_{h}$ in the subproblem $\left.G\right|_{h}$ in a natural way: for any $k^{\prime} \geq k$ and any sequence $\left(a_{k+1}, a_{k+2}, \ldots, a_{k^{\prime}}\right)$ of actions,

$$
\left.b\right|_{h}\left(a_{k+1}, a_{k+2}, \ldots, a_{k^{\prime}}\right)=b\left(h, a_{k+1}, a_{k+2}, \ldots, a_{k^{\prime}}\right) .
$$

The strategy $\left.b\right|_{h}$ is often called the continuation strategy of $b$ at history $h$. Thus, for every behavior strategy $b$, an $\mathcal{R}^{\mathrm{DS}}$-specification $\psi$ defines a charge $\psi\left(\left.b\right|_{h}\right) \in \mathcal{C}\left(P, \mathcal{R}^{\mathrm{DS}}\right)$.

Lemma 4. There exists a unique $\mathcal{R}^{\mathrm{DS}}$-specification $\psi$ with the following property: for every behavior strategy $b$, for every history $h$, and for every $Q \in \mathcal{R}^{\mathrm{DS}}$,

$$
\psi\left(\left.b\right|_{h}\right)\left(\left.Q\right|_{h}\right)=\int_{a \in A} \psi\left(\left.b\right|_{h a}\right)\left(\left.Q\right|_{h a}\right) b(h)(\mathrm{d} a) .
$$

So, there is a unique $\mathcal{R}^{\mathrm{DS}}$-specification $\psi$ such that, for each behavior strategy $b$, history $h$, action $a$, and for every clopen set, the corresponding probability under $\psi\left(\left.b\right|_{h}\right)$ should be equal to the expectation of the probability at the next period under $\psi\left(\left.b\right|_{h a}\right)$. This lemma is essentially Theorem 2.8.1 in Dubins and Savage (2014); Theorem 4.1 in Purves and Sudderth (1976) is also similar.

We define $\psi^{\mathrm{DS}}$ to be the unique $\mathcal{R}^{\mathrm{DS}}$-specification in Lemma 4 . Note that by the definition of an $\mathcal{R}^{\mathrm{DS}}$-specification, we have for every behavior strategy $b$ and every $Q \in \mathcal{R}^{\mathrm{f}}$ that

$$
\psi^{\mathrm{DS}}(b)(Q)=c^{\mathrm{f}}(b)(Q) .
$$

The next lemma conveys a useful property of the $\mathcal{R}^{\mathrm{DS}}$-specification $\psi^{\mathrm{DS}}$. The proof can be found in the Appendix.

Lemma 5. Consider a behavior strategy $b$ and let $Q$ be a nonempty element of $\mathcal{R}^{\mathrm{DS}}$. Assume that for every history $h \in H$ for which $\left.Q\right|_{h} \neq \varnothing$ we have

$$
b(h)\left(\left\{a \in A:\left.Q\right|_{(h, a)} \neq \varnothing\right\}\right)=1 .
$$

Then $\psi^{\mathrm{DS}}(b)(Q)=1$.

The above lemma has the following meaning. Consider a nonempty clopen set $Q$ of plays. A history $h$ is consistent with $Q$ if $Q$ contains a play that starts with $h$, i.e. $\left.Q\right|_{h} \neq \varnothing$. Consider a behavior strategy $b$. Assume that whenever a history $h$ is consistent with $Q$, then the charge $b(h)$ places probability 1 on those actions that keep the history consistent with $Q$. Then, the set $Q$ has probability 1 under $b$, with respect to the $\mathcal{R}^{\mathrm{DS}}$-specification $\psi^{\mathrm{DS}}$.

\subsection{Equivalence from mixed strategies to behavior strategies}

In this subsection we analyze when a mixed strategy has an equivalent behavior strategy within the setting of the previous subsection.

We already know from Theorem 3 that when the action space is infinite, there is a mixed strategy without an $\left(\mathcal{R}^{\mathrm{DS}}, \psi^{\mathrm{DS}}\right)$-equivalent behavior strategy. In contrast, when the action space is finite, we obtain a positive result. 
Theorem 4. Suppose that the action space A is finite. Then for every mixed strategy there exists an $\left(\mathcal{R}^{\mathrm{DS}}, \psi^{\mathrm{DS}}\right)$-equivalent behavior strategy.

Proof. Take a mixed strategy $m$. Let $H_{m}$ denote the set of histories $h$ that are reached with positive probability under $m$, i.e. $m^{*}([h])>0$.

We define a behavior strategy $b$. Take a history $h \in H$. If $h \in H \backslash H_{m}$, then $b(h)$ is an arbitrary charge on $\left(A, 2^{A}\right)$. If $h \in H_{m}$, then define $b(h)$ by for every $a \in A$ letting

$$
b(h)(a)=\frac{m^{*}([h, a])}{m^{*}([h])} .
$$

Now it follows for any history $h=\left(a_{1}, \ldots, a_{n}\right)$, by induction on the length $n$ of the history, that

$$
m^{*}([h])=\psi^{\mathrm{DS}}(b)([h])=b(\varnothing)\left(a_{1}\right) \cdots b\left(a_{1}, \ldots, a_{n-1}\right)\left(a_{n}\right) .
$$

Since the action space $A$ is finite, this implies directly that $m^{*}(Q)=\psi^{\mathrm{DS}}(b)(Q)$ for every $Q \in R^{\mathrm{f}}$. Because $\mathcal{R}^{\mathrm{f}}=\mathcal{R}^{\mathrm{DS}}$ by Lemma 3 , the behavior strategy $b$ is $\left(\mathcal{R}^{\mathrm{DS}}, \psi^{\mathrm{DS}}\right)$-equivalent to $m$.

\subsection{Existence of an optimal behavior strategy}

We now prove that, in the approach of Dubins and Savage, an optimal behavior strategy always exists provided that the payoff function is integrable.

Theorem 5. For every $\mathcal{R}^{\mathrm{DS}}$-integrable payoff function $u$, there exists an $\left(\mathcal{R}^{\mathrm{DS}}, \psi^{\mathrm{DS}}\right)$-optimal behavior strategy.

Proof. We begin by giving the idea of the proof. Suppose that the payoff function $u$ is $\mathcal{R}^{\mathrm{DS}}$-integrable. Let $U^{*}=\sup _{p \in P} u(p)$. Without being precise, the main idea is to construct a sequence of clopen sets $B_{1} \supseteq B_{2} \supseteq B_{3} \supseteq \cdots$ of plays such that the best value of $u$ on $B_{n}$ converges to $U^{*}$ :

$$
\lim _{n \rightarrow \infty} \sup _{p \in B_{n}} u(p)=U^{*} .
$$

Let us say that a history $h$ is consistent with $B_{n}$ if $B_{n}$ contains a play that starts with $h$. This means that at $h$ we still have the possibility to stay in $B_{n}$.

We then construct a behavior strategy $b$ that behaves as follows. Consider a history $h$. There are three cases. If $h$ is not consistent with any $B_{n}$, then $b(h)$ is arbitrary. If $h$ is consistent with $B_{1}, \ldots, B_{n}$ but not with $B_{n+1}, B_{n+2}, \ldots$, then $b(h)$ places probability 1 on an action that keeps consistency with $B_{n}$. Finally, if $h$ is consistent with every $B_{n}$, then $b(h)$ intuitively recommends choosing a natural number $n$ according to a diffuse charge on $\left(\mathbb{N}, 2^{\mathbb{N}}\right)$ and then playing an action that keeps consistency with $B_{n}$. So, the idea of the behavior strategy $b$ is to stay consistent with $B_{n}$ for as large values of $n$ as possible. It will then follow from (5) that $b$ is $\left(\mathcal{R}^{\mathrm{DS}}, \psi^{\mathrm{DS}}\right)$-optimal, as desired.

The formal proof consists of four steps.

Step 1. In this part of the proof we construct a number of objects:

(i) For every $n \in \mathbb{N}$, we construct an $\mathcal{R}^{\mathrm{DS}}$-measurable simple function $u_{n}$ of the form

$$
u_{n}(p)=\sum_{B \in \mathcal{B}_{n}} c_{B} \cdot \mathbb{I}_{B}(p),
$$


where $c_{B} \in \mathbb{R}$ for every $B \in \mathcal{B}_{n}$ and $\mathcal{B}_{n} \subseteq \mathcal{R}^{\mathrm{DS}}$ is a finite partition of $P$. Our construction guarantees that the following properties hold for every $n \in \mathbb{N}$ : (i.1) $u_{n} \leq u$; (i.2) $\sup _{p \in P}\left|u_{n}(p)-u(p)\right| \leq \frac{1}{n}$ for every play $p \in P$; and (i.3) the partition $\mathcal{B}_{n+1}$ is (weakly) finer than the partition $\mathcal{B}_{n}$ : every set in $\mathcal{B}_{n+1}$ is a subset of a set in $\mathcal{B}_{n}$.

(ii) For every $n \in \mathbb{N}$ we identify a set $B_{n} \in \mathcal{B}_{n}$ such that (ii.1) for every $n \in \mathbb{N}, B_{n} \supseteq$ $B_{n+1}$; (ii.2) $c_{B_{n}}$ converges to $U^{*}$ as $n$ tends to infinity.

Construction for (i): The function $u$ is $\mathcal{R}^{\mathrm{DS}}$-integrable. Hence, as discussed in Section 2, for every $n \in \mathbb{N}$ there exists an $\mathcal{R}^{\mathrm{DS}}$-measurable simple function $v_{n}$ such that $\sup _{p \in P}\left|v_{n}(p)-u(p)\right| \leq \frac{1}{2 n}$ for every play $p \in P$. Let $u_{n}(p)=v_{n}(p)-\frac{1}{2 n}$ for every play $p \in P$. Then, for every $n \in \mathbb{N}, u_{n}$ is an $\mathcal{R}^{\mathrm{DS}}$-measurable simple function such that $u_{n} \leq u$ and $\sup _{p \in P}\left|u_{n}(p)-u(p)\right| \leq \frac{1}{n}$ for every play $p \in P$. So, (i.1) and (i.2) hold. For each $n \in \mathbb{N}$, let $\mathcal{B}_{n} \subseteq \mathcal{R}^{\mathrm{DS}}$ be the partition of the set $P$ of plays corresponding to $u_{n}$. By splitting sets in $\mathcal{B}_{2}$ if necessary, we can achieve that $\mathcal{B}_{2}$ is (weakly) finer than the partition $\mathcal{B}_{1}$. Next, by splitting sets in $\mathcal{B}_{3}$ if necessary, we can achieve that $\mathcal{B}_{3}$ is (weakly) finer than the partition $\mathcal{B}_{2}$. By continuing this way, we can make sure that (i.3) holds as well.

Construction for (ii): For every $n \in \mathbb{N}$ let the play $p_{n} \in P$ be such that $u\left(p_{n}\right) \geq U^{*}-\frac{1}{n}$. Because the partition $\mathcal{B}_{1}$ is finite, there exists a set $B_{1} \in \mathcal{B}_{1}$ such that the set $\left\{m \in \mathbb{N}: p_{m} \in\right.$ $\left.B_{1}\right\}$ is infinite. Because $\mathcal{B}_{2}$ is finer than $\mathcal{B}_{1}$, there also exists a set $B_{2} \in \mathcal{B}_{2}$ such that $B_{2} \subseteq B_{1}$ and the set $\left\{m \in \mathbb{N}: p_{m} \in B_{2}\right\}$ is infinite. Continuing in this way we define $B_{n}$ for each $n \in \mathbb{N}$, and then (ii.1) holds. Now assume that $p_{m} \in B_{n}$ for some $m, n \in \mathbb{N}$. Then, by the choice of the play $p_{m}$, by (i.2), and by $p_{m} \in B_{n}$, we have

$$
U^{*}-\frac{1}{m} \leq u\left(p_{m}\right) \leq u_{n}\left(p_{m}\right)+\frac{1}{n}=c_{B_{n}}+\frac{1}{n} .
$$

As the set $\left\{m \in \mathbb{N}: p_{m} \in B_{n}\right\}$ is infinite, the inequality (6) holds for infinitely many $m \in \mathbb{N}$, and hence

$$
U^{*} \leq c_{B_{n}}+\frac{1}{n}
$$

This implies that (ii.2) holds as well.

Step 2. In this step we construct a behavior strategy $b$. First, we need some notation. Take a history $h \in H$. Let

$$
N_{h}^{*}=\left\{n \in \mathbb{N}:[h] \cap B_{n} \neq \varnothing\right\} .
$$

Intuitively, $n \in N_{h}^{*}$ means that the history $h$ is consistent with the set $B_{n}$ in the sense that there is a play in $B_{n}$ that extends $h$. More precisely, if $n \in N_{h}^{*}$, then there is a play $p_{h}^{n} \in \mathcal{B}_{n}$ such that $h \prec p_{h}^{n}$, and hence there is an action $a_{h}^{n} \in A$, i.e. the action in $p_{h}^{n}$ after $h$, such that $\left(h, a_{h}^{n}\right) \prec p_{h}^{n}$. Notice the following properties for any history $h \in H$ :

(a) If $m \in N_{h}^{*}$ and $n \leq m$, then $n \in N_{h}^{*}$ and $n \in N_{\left(h, a_{h}^{m}\right)}^{*}$. We prove this property.

Assume $m \in N_{h}^{*}$ and $n \leq m$. Then, by (ii.1), $[h] \cap \mathcal{B}_{n} \supseteq[h] \cap \mathcal{B}_{m} \neq \varnothing$, and hence $n \in N_{h}^{*}$.

Further, as $m \in N_{h}^{*}$, we have $p_{h}^{m} \in\left[\left(h, a_{h}^{m}\right)\right] \cap \mathcal{B}_{m}$, which implies that $\left[\left(h, a_{h}^{m}\right)\right] \cap$ $\mathcal{B}_{m} \neq \varnothing$. Hence, $m \in N_{\left(h, a_{h}^{m}\right)}^{*}$. By the previous part, $n \in N_{\left(h, a_{h}^{m}\right)}^{*}$. 
(b) We have one of the following three cases: $N_{h}^{*}$ is empty, or $N_{h}^{*}=\{1, \ldots, m\}$ for some $m \in \mathbb{N}$, or $N_{h}^{*}=\mathbb{N}$. This follows from property (a).

Now we define a behavior strategy $b$. Take an arbitrary history $h \in H$. We need to define the charge $b(h) \in \mathcal{C}\left(A, 2^{A}\right)$. Based on property (b), we distinguish three different cases. If $N_{h}^{*}$ is empty, then let $b(h)$ be an arbitrary charge on the action space. If $N_{h}^{*}=\{1, \ldots, m\}$ for some $m \in \mathbb{N}$, then define $b(h)$ to be the Dirac charge on $a_{h}^{m}$. That is, for every set $B \subseteq A$, we have $b(h)(B)=1$ if $a_{h}^{m} \in B$ and $b(h)(B)=0$ if $a_{h}^{m} \notin B$. Finally, if $N_{h}^{*}=\mathbb{N}$, then let $\tau$ be a diffuse charge on $\left(\mathbb{N}, 2^{\mathbb{N}}\right)$, and define $b(h)$ by for each $A^{\prime} \subseteq A$ letting

$$
b(h)\left(A^{\prime}\right)=\tau\left(\left\{n \in \mathbb{N}: a_{h}^{n} \in A^{\prime}\right\}\right) .
$$

Intuitively, in this final case $b(h)$ recommends choosing a natural number $n$ according to $\tau$ and then playing the action $a_{h}^{n}$. So, in all three cases the idea of the behavior strategy $b$ is to stay consistent with $B_{n}$ for as large values of $n$ as possible.

Step 3. We show that $\psi^{\mathrm{DS}}(b)\left(B_{n}\right)=1$ for every $n \in \mathbb{N}$. Take $n \in \mathbb{N}$. We prove this by applying Lemma 5. Let $Q=B_{n}$. We already know that $Q$ is clopen, as $Q=B_{n} \in \mathcal{B}_{n} \subseteq \mathcal{R}^{\mathrm{DS}}$. Take a history $h \in H$, and assume that $\left.Q\right|_{h} \neq \varnothing$. Then there is a play $p \in Q$ such that $p \succ h$. Thus, $n \in N_{h}^{*} \neq \varnothing$.

In view of property (b), we have two cases. Assume first that $N_{h}^{*}=\{1, \ldots, m\}$ for some $m \in \mathbb{N}$. Obviously, $m \geq n$. By property (a), $n \in N_{\left(h, a_{h}^{m}\right)}^{*}$. Thus, $\left.Q\right|_{\left(h, a_{h}^{m}\right)} \neq \varnothing$. Recall that $b(h)$ is the Dirac charge on $a_{h}^{m}$ in this case, so $b$ satisfies the condition of Lemma 5.

Assume next that $N_{h}^{*}=\mathbb{N}$. For every $m \geq n$, by property (a), $n \in N_{\left(h, a_{h}^{m}\right)}^{*}$ and hence $\left.Q\right|_{\left(h, a_{h}^{m}\right)} \neq \varnothing$. As $b(h)\left(\left\{a_{h}^{n}, a_{h}^{n+1}, \ldots\right\}\right)=1$, the strategy $b$ satisfies the condition of Lemma 5 in this case as well.

Thus, by Lemma $5, \psi^{\mathrm{DS}}(b)(Q)=1$, i.e. $\psi^{\mathrm{DS}}(b)\left(B_{n}\right)=1$, as desired.

Step 4. We show that the behavior strategy $b$ is $\left(\mathcal{R}^{\mathrm{DS}}, \psi^{\mathrm{DS}}\right)$-optimal. By (i.1) and by Step 3, we have for every $n \in \mathbb{N}$ that

$$
\int_{p \in P} u(p) \psi^{\mathrm{DS}}(b)(\mathrm{d} p) \geq \int_{p \in P} u_{n}(p) \psi^{\mathrm{DS}}(b)(\mathrm{d} p)=c_{B_{n}} .
$$

Hence, by (ii.2),

$$
\int_{p \in P} u(p) \psi^{\mathrm{DS}}(b)(\mathrm{d} p)=U^{*}
$$

This completes the proof.

\section{Extension of the Dubins and Savage approach to larger algebras}

In the previous section we considered the classical approach by Dubins and Savage based on the algebra of clopen sets of plays. There have been several attempts to extend this algebra so that a probability can be assigned to a larger class of sets under every behavior strategy. One common aspect of these extensions is to include each singleton play.

One essential motivation to consider algebras that contain singleton plays is the following. Take any play $p$ and a behavior strategy that tries to induce the play $p$ by choosing the corresponding actions with probability 1 . Since the set $\{p\}$ is not open, it is not clopen either, and hence in the approach of Dubins and Savage we do not assign a probability to the set 
$\{p\}$. Therefore, we cannot say that the behavior strategy induces $p$ with probability 1 . This is, of course, fully acceptable in the context of finite additivity, as implementing $b$ involves successive usage of an infinite sequence of charges. Yet, it is certainly natural to extend the algebra so that it includes all singleton plays and define the specification in such a way that the behavior strategy above induces probability 1 on the set $\{p\}$. Clearly, this brings us a step closer to countable additivity.

Consider a competent algebra $\mathcal{P}$ on the set $P$ of plays with $\{p\} \in \mathcal{P}$ for every play $p \in P$. We say that a $\mathcal{P}$-specification $\psi^{\mathcal{P}}$ respects singleton plays if for each behavior strategy $b$ and each play $p=\left(a_{1}, a_{2}, \ldots\right)$ we have

$$
\psi^{\mathcal{P}}(b)(\{p\})=\prod_{n=1}^{\infty} b\left(a_{1}, \ldots, a_{n-1}\right)\left(a_{n}\right) .
$$

In this section, first we examine when a mixed strategy has an equivalent behavior strategy with respect to a competent algebra that contains all singleton plays and a specification that respects singleton plays. Second, we investigate the existence of an optimal behavior strategy.

\subsection{Equivalence from mixed strategies to behavior strategies}

We first demonstrate that even if the action space is finite, there is a mixed strategy $m$ that has no $\left(\mathcal{P}, \psi^{\mathcal{P}}\right)$-equivalent behavior strategy for any competent algebra $\mathcal{P}$ on the set $P$ of plays that contains all singleton plays and any $\mathcal{P}$-specification $\psi^{\mathcal{P}}$ that respects singleton plays.

Example 1. Consider a decision problem with action space $A=\{c, s\}$. One can think of it as a stopping problem, with $c$ standing for 'continue' and $s$ standing for 'stop'. For every $n \in \mathbb{N}$, let $f_{n}$ be a pure strategy that prescribes action $c$ at all periods before $n$ and action $s$ at period $n$. Let $\tau$ be a diffuse charge on $\left(\mathbb{N}, 2^{\mathbb{N}}\right)$. Let the mixed strategy $m$ be defined by $m(G)=\tau(\{n \in$ $\left.\mathbb{N}: f_{n} \in G\right\}$ ) for all $G \subseteq F$. Thus, intuitively, $m$ prescribes choosing $n \in \mathbb{N}$ according to $\tau$ and then playing the pure strategy $f_{n}$. This means that if the decision maker plays according to $m$, then they eventually play $s$ with probability 1 , but the probability that $s$ is played in the first $T$ periods is 0 for every $T \in \mathbb{N}$. This construction is similar to the optimal mixed strategy in the proof of Theorem 1.

This mixed strategy $m$ has no $\left(\mathcal{P}, \psi^{\mathcal{P}}\right)$-equivalent behavior strategy for any competent algebra $\mathcal{P}$ on the set $P$ of plays that contains all singleton plays and any $\mathcal{P}$-specification $\psi^{\mathcal{P}}$ that respects singleton plays. A precise argument follows from the proof of Theorem 6 . Here we only provide the intuition.

Let $b$ be a behavior strategy. Also, for every $n \in \mathbb{N}$, let $c^{n}$ denote the history $(c, \ldots, c)$ of length $n$, and let $c^{\infty}$ denote the play only having action $c$. As the probability under $m$ that the decision maker plays action $s$ in the first $T$ periods is 0 for every $T \in \mathbb{N}$, if $b\left(c^{n}\right)$ does not put probability 1 on action $c$ for some $n \in N$ then $b$ cannot be $\left(\mathcal{P}, \psi^{\mathcal{P}}\right)$-equivalent to $m$. So, assume that $b\left(c^{n}\right)$ puts probability 1 on action $c$ for all $n \in N$. Then, as the singleton $\left\{c^{\infty}\right\}$ is contained in the algebra $\mathcal{P}$, by (7) we have $\psi(b)\left(\left\{c^{\infty}\right\}\right)=1$. Because $m^{*}\left(\left\{c^{\infty}\right\}\right)=0$, also in this case $b$ cannot be $\left(\mathcal{P}, \psi^{\mathcal{P}}\right)$-equivalent to $m$.

The following theorem is based on Example 1.

Theorem 6. Let $\mathcal{P}$ be a competent algebra on the set $P$ of plays containing all singleton plays, and let $\psi^{\mathcal{P}}$ be a $\mathcal{P}$-specification that respects singleton plays. Then there exists a mixed strategy that has no $\left(\mathcal{P}, \psi^{\mathcal{P}}\right)$-equivalent behavior strategy. 
Proof. Let $a$ and $a^{\prime}$ denote two different actions in $A$. For every $n \in \mathbb{N}$, let $p_{n}$ denote the play that has action $a$ up to period $n$ and has action $a^{\prime}$ from period $n+1$ onwards. The sequence of plays $\left(p_{n}\right)_{n \in \mathbb{N}}$ converges to the play $p=(a, a, \ldots)$.

For all $n \in \mathbb{N}$, let $f_{n}$ denote a pure strategy that induces the play $p_{n}$. Let $\tau$ be a diffuse charge on $\left(\mathbb{N}, 2^{\mathbb{N}}\right)$. Now consider the mixed strategy $m$ that, intuitively, first chooses a natural number $n \in \mathbb{N}$ according to $\tau$ and then plays the pure strategy $f_{n}$. Formally, for every subset $F^{\prime}$ of the set $F$ of pure strategies we have

$$
m\left(F^{\prime}\right)=\tau\left(\left\{n \in \mathbb{N} \mid f_{n} \in F^{\prime}\right\}\right) .
$$

Hence, for every $P^{\prime} \subseteq P$,

$$
m^{*}\left(P^{\prime}\right)=\tau\left(\left\{n \in \mathbb{N} \mid p_{n} \in P^{\prime}\right\}\right) .
$$

We show that the mixed strategy $m$ has no $\left(\mathcal{P}, \psi^{\mathcal{P}}\right)$-equivalent behavior strategy. Indeed, take any behavior strategy $b$. We distinguish two cases.

First, assume that there is a history of the form $h=(a, \ldots, a) \prec p$ such that the charge $b(h)$ places probability less than 1 on action $a$. We can assume that $h$ is the shortest history with this property. Let $Q$ be the set of all histories of the form $(h, x)$ where $x \in A \backslash\{a\}$. The set $[Q]$ belongs to the algebra $\mathcal{R}^{\mathrm{f}} \subseteq \mathcal{P}$, and

$$
\psi^{\mathcal{P}}(b)([Q])=c^{\mathrm{f}}(b)([Q])=1-b(h)(a)>0 .
$$

This is, however, in contradiction with $m^{*}([Q])=0$.

Now assume that for every history of the form $h=(a, \ldots, a) \prec p$, the charge $b(h)$ places probability 1 on action $a$. As $\psi^{\mathcal{P}}$ respects singleton plays, $\psi^{\mathcal{P}}(b)(\{p\})=1$. This contradicts $m^{*}(\{p\})=0$.

Thus, the mixed strategy $m$ has no $\left(\mathcal{P}, \psi^{\mathcal{P}}\right)$-equivalent behavior strategy.

Theorem 6 applies to several algebras that have been previously considered in the literature. For example, Dubins (1974) considered the algebra generated by open sets, and Purves and Sudderth (1976) considered the Borel sigma-algebra. Part of the authors' motivation concerns stating finitely additive generalizations of classical probabilistic limit laws in the usual fashion.

Both the previously mentioned algebras are competent and contain all singletons, and their corresponding specifications respect singleton plays. In the remainder of this subsection we show that Theorem 6 applies to the algebra generated by the open sets and the corresponding specification.

Given a charge $\mu$ on the clopen subsets of $P$, Dubins (1974) defined a probability for each open subset of $P$ through inner approximations by clopen sets. That is, if $O \subseteq P$ is open, then he let $\mu^{\prime}(O)$ be the supremum of $\mu(Z)$ where $Z \subseteq O$ is clopen. His Theorem 2 showed that this mapping $\mu^{\prime}$ defined on the open sets has a unique extension to a charge on the whole algebra generated by the open sets.

By translating the result of Dubins (1974), we now define a competent algebra and a specification. For every behavior strategy $b$ and open set $O \subseteq P$ we let

$$
c^{\mathrm{O}}(b)(O)=\sup _{Z \in \mathcal{R}^{\mathrm{DS}}, Z \subseteq O} \psi^{\mathrm{DS}}(b)(Z) .
$$

Let $\mathcal{R}^{\circ}$ be the algebra generated by the open subsets of $P$. That is, $\mathcal{R}^{\circ}$ is the smallest algebra of subsets of $P$ that contains all open sets. By Lemma $3, \mathcal{R}^{\mathrm{f}} \subseteq \mathcal{R}^{\mathrm{DS}} \subset \mathcal{R}^{\mathrm{o}}$. So, in particular, $\mathcal{R}^{\mathrm{o}}$ is competent. 
By the result of Dubins (1974), for every behavior strategy $b$ there is a unique charge $\psi^{\circ}(b)$ on the algebra $\mathcal{R}^{0}$ such that, for every open set $O \subseteq P$,

$$
\psi^{\mathrm{o}}(b)(O)=c^{\mathrm{o}}(b)(O) .
$$

Observe the following. If $Q \in \mathcal{R}^{\mathrm{f}}$ then $Q \in \mathcal{R}^{\mathrm{DS}}$, and hence in view of (9), (8), and (4) we have for every behavior strategy $b$ that

$$
\psi^{\mathrm{o}}(b)(Q)=c^{\mathrm{o}}(b)(Q)=\psi^{\mathrm{DS}}(b)(Q)=c^{\mathrm{f}}(b)(Q) .
$$

This means that $\psi^{\mathrm{o}}$ is an $\mathcal{R}^{\mathrm{o}}$-specification.

Lemma 6. The algebra $\mathcal{R}^{\circ}$ contains all singleton plays, and the $\mathcal{R}^{\mathrm{o}}$-specification $\psi^{\mathrm{o}}$ respects singleton plays.

Proof. Since each singleton $\{p\}$, where $p \in P$, is closed, $\mathcal{R}^{\circ}$ contains all singleton plays.

We now show that $\psi^{\circ}$ respects singleton plays. Let $b$ be a behavior strategy and let $p=$ $\left(a_{1}, a_{2}, \ldots\right) \in P$. For each $n \in \mathbb{N}$, let $H_{n} \subset A^{n}$ denote the set of histories at period $n$ that follow $p$ until period $n-1$ and then deviate from $a_{n}$ at period $n$ :

$$
H_{n}=\left\{\left(a_{1}, \ldots, a_{n-1}, a\right): a \neq a_{n}\right\},
$$

and let $Q_{n}=H_{1} \cup \cdots \cup H_{n}$. For each $n \in \mathbb{N}$, we have $Q_{n} \in \mathcal{R}^{\mathrm{f}}$, and hence, by (10),

$$
\psi^{\mathrm{o}}(b)\left(Q_{n}\right)=c^{\mathrm{f}}(b)\left(Q_{n}\right)=1-c^{\mathrm{f}}(b)\left(\left[a_{1}, \ldots, a_{n}\right]\right)=1-\prod_{k=1}^{n} b\left(a_{1}, \ldots, a_{k-1}\right)\left(a_{k}\right) .
$$

Now take any clopen set $Q \subset P \backslash\{p\}$. It is easy to see that there is $n \in \mathbb{N}$ such that $Q \subseteq Q_{n}$. Indeed, otherwise $p$ would be a limit point of $Q$, which is impossible. As $Q_{n} \subset P \backslash\{p\}$, we obtain

$$
\sup _{Q \in \mathcal{R}^{\mathrm{DS}}, Q \subseteq P \backslash\{p\}} \psi^{\mathrm{o}}(Q)=\sup _{n \in \mathbb{N}} \psi^{\mathrm{o}}\left(Q_{n}\right)=1-\prod_{k=1}^{\infty} b\left(a_{1}, \ldots, a_{k-1}\right)\left(a_{k}\right) .
$$

Since $P \backslash\{p\}$ is open, by (9) and (8) we have

$$
\psi^{\mathrm{o}}(P \backslash\{p\})=1-\prod_{k=1}^{\infty} b\left(a_{1}, \ldots, a_{k-1}\right)\left(a_{k}\right),
$$

implying

$$
\psi^{\mathrm{o}}(\{p\})=\prod_{k=1}^{\infty} b\left(a_{1}, \ldots, a_{k-1}\right)\left(a_{k}\right) .
$$

Thus, $\psi^{\mathrm{o}}(b)$ respects singleton plays.

The next theorem follows from Lemma 6 and Theorem 6.

Theorem 7. In any decision problem there exists a mixed strategy that has no $\left(\mathcal{R}^{\mathrm{o}}, \psi^{\mathrm{o}}\right)$ equivalent behavior strategy. 
We emphasize that the specification $\psi^{0}$ defines a charge on $\mathcal{R}^{\circ}$ for all behavior strategies. Given a specific behavior strategy $b$, Purves and Sudderth (1976) extended the charge $\psi^{\circ}(b)$ from the algebra $\mathcal{R}^{\circ}$ to $\mathcal{A}(b)$. The algebra $\mathcal{A}(b)$ contains the sets of plays which can be approximated by closed sets from the inside and open sets from the outside in such a way that the $\psi^{\mathrm{o}}(b)$-probability of their difference can be made arbitrarily small (see their Proposition 2.1). Their Theorem 5.1 showed that all elements of the Borel sigma-algebra on $P$ can be approximated this way, so the Borel sigma-algebra is a subset of $\mathcal{A}(b)$. This means that for every behavior strategy $b$ there is a charge defined on the Borel sigma-algebra which agrees with $\psi^{\mathrm{o}}(b)$ on the algebra $\mathcal{R}^{\mathrm{o}}$.

\subsection{Existence of an optimal behavior strategy}

The following theorem is in contrast with Theorem 5 that we obtained under the approach of Dubins and Savage.

Theorem 8. Let $\mathcal{P}$ be a competent algebra on the set $P$ of plays containing all singleton plays, and let $\psi^{\mathcal{P}}$ be a $\mathcal{P}$-specification that respects singleton plays. Then there exists a $\mathcal{P}$-integrable payoff function $u$ for which there is no $\left(\mathcal{P}, \psi^{\mathcal{P}}\right)$-optimal behavior strategy.

Proof. Take an action $a \in A$. Let the payoff function $u$ be defined as follows: if the decision maker chooses an action other than $a$ at period $n$ for the first time, then the payoff is $\frac{n}{n+1}$; if they choose action $a$ at all periods, then the payoff is 0 . More precisely, for every $n \in \mathbb{N}$, let $H_{n}$ denote the histories of length $n$ of the form $\left(a, \ldots, a, a^{\prime}\right)$, where $a^{\prime} \in A \backslash\{a\}$. Then $u(p)=\frac{n}{n+1}$ if $p \in\left[H_{n}\right]$ for some $n \in \mathbb{N}$, and $u(a, a, \ldots)=0$.

We show that $u$ is $\mathcal{P}$-integrable by proving that for every $\varepsilon>0$ there exists a $\mathcal{P}$-measurable simple function $u^{\prime}: P \rightarrow \mathbb{R}$ such that $\left|u(p)-u^{\prime}(p)\right| \leq \varepsilon$ for every $p \in P$. Indeed, take $\varepsilon>0$, and let $m \in \mathbb{N}$ such that $m \geq \frac{1-\varepsilon}{\varepsilon}$. Consider the partition of $P$ consisting of the following sets:

$$
\left[H_{1}\right],\left[H_{2}\right], \ldots,\left[H_{m-1}\right], \bigcup_{k \geq m}\left[H_{m}\right] \backslash\{(a, a, \ldots)\},\{(a, a, \ldots)\} \text {. }
$$

Since each $\left[H_{n}\right]$ belongs to $\mathcal{R}^{\mathrm{f}}$, and since the algebra $\mathcal{P}$ is competent and contains all singleton plays (in particular it contains $\{(a, a, \ldots)\})$, we can conclude that all these sets belong to $\mathcal{P}$. Define the following function $u^{\prime}: P \rightarrow \mathbb{R}$. Let $u^{\prime}(p)=u(p)=\frac{n}{n+1}$ if $p \in\left[H_{n}\right]$ for some $n \leq m-$ 1 , let $u^{\prime}(p)=1$ for all $p \in \cup_{k \geq m}\left[H_{m}\right] \backslash\{(a, a, \ldots)\}$, and let $u^{\prime}(a, a, \ldots)=u(a, a, \ldots)=0$. It follows that $u^{\prime}$ is a $\mathcal{P}$-measurable simple function such that $\left|u(p)-u^{\prime}(p)\right| \leq \varepsilon$ for every $p \in P$.

It follows similarly to the proof of Theorem 6 that there is no behavior strategy $b$ such that $U\left(b \mid \mathcal{P}, \psi^{\mathcal{P}}\right)=1$. This means that there is no $\left(\mathcal{P}, \psi^{\mathcal{P}}\right)$-optimal behavior strategy.

\section{Concluding remarks}

In a class of finitely additive decision problems, we examined equivalence between mixed and behavior strategies, in particular for optimal strategies. Under the classical approach of Dubins and Savage (2014), we proved that: (1) if the action space is finite, every mixed strategy has an equivalent behavior strategy, and (2) even if the action space is infinite, at least one optimal mixed strategy has an equivalent behavior strategy. The Dubins and Savage approach turned out to be essentially maximal: these two statements are no longer valid if we take any extension of the algebra considered by Dubins and Savage that includes all singleton plays. Our results suggest that mixed strategies may be more suitable for studying finitely additive decision problems. 
An interesting question would be to look at hybrid strategies, in which in certain parts of the decision tree mixed strategies are used and in other parts of the decision tree behavior strategies are used. For example, such a strategy could prescribe following a behavior strategy up to a certain period and then, according to a mixed strategy, choosing a pure continuation strategy for the remaining part of the decision problem.

Another interesting question is how to deal with decision problems in which multiple decision makers are involved. These problems are substantially more difficult. Suppose that there are two decision makers who use mixed strategies $m_{1}$ and $m_{2}$ respectively. The first question is which charge arises under $\left(m_{1}, m_{2}\right)$ on the set of pure strategy pairs $\left(f_{1}, f_{2}\right)$. The pair $\left(m_{1}, m_{2}\right)$ naturally induces the probability $m_{1}\left(F_{1}^{\prime}\right) \cdot m_{2}\left(F_{2}^{\prime}\right)$ on each rectangle $F_{1}^{\prime} \times F_{2}^{\prime}$, where $F_{1}^{\prime}$ and $F_{2}^{\prime}$ are subsets of the sets $F_{1}$ and $F_{2}$ of pure strategies for the two decision makers. The probabilities on rectangles generally do not have a unique extension to a charge on the power set of $F_{1} \times F_{2}$. Consequently, the expected payoff under $\left(m_{1}, m_{2}\right)$ is not always uniquely defined. For further details, we refer to Flesch et al. (2017) and the references therein.

We finally remark that in decision problems in which chance moves are present we can model chance as another, but non-strategic, decision maker who chooses their actions according to a fixed probabilistic rule. Therefore, in such decision problems, similar questions arise.

\section{Appendix A}

Proof of Theorem 2. Let $\mathcal{P}$ be an algebra of $P$, and $\psi^{\mathcal{P}}$ be a $\mathcal{P}$-specification. Consider a behavior strategy $b$. Take an arbitrary charge $B \in \mathcal{C}\left(P, 2^{P}\right)$ that extends the charge $\psi^{\mathcal{P}}(b) \in$ $\mathcal{C}(P, \mathcal{P})$. As explained in Section 2, such an extension exists. Let $\phi: P \rightarrow F$ be a function such that if $\phi(p)=f$ then $p_{f}=p$. So, $\phi(p)$ is a pure strategy that induces the play $p$.

For a set $G \subseteq F$, define

$$
m(G)=B\left(\left\{p_{f}: f \in G \cap \phi(P)\right\}\right) .
$$

Intuitively, $m(G)$ is the probability under $B$ of the set of plays that are induced by a pure strategy in $G \cap \phi(P)$. One can check that $m$ is a mixed strategy.

Finally, take a set $Q \in \mathcal{P}$. Let $G=\left\{f \in F: p_{f} \in Q\right\}$. Then $\left\{p_{f}: f \in G \cap \phi(P)\right\}=Q$. In view of (1), we have

$$
m^{*}(Q)=m(G)=B\left(\left\{p_{f}: f \in G \cap \phi(P)\right\}\right)=B(Q) .
$$

Thus, the strategies $m$ and $b$ are $\left(\mathcal{P}, \psi^{\mathcal{P}}\right)$-equivalent.

Proof of Lemma 5. Consider a behavior strategy $b$ and let $Q$ be a nonempty element of $\mathcal{R}^{\mathrm{DS}}$ such that for every history $h \in H$ for which $\left.Q\right|_{h} \neq \varnothing$ we have

$$
b(h)\left(\left\{a \in A:\left.Q\right|_{(h, a)} \neq \varnothing\right\}\right)=1 .
$$

Assume by way of contradiction that $d:=\psi^{\mathrm{DS}}(b)(Q)<1$. Then by (11) and Lemma 4 there is an action $a_{1} \in A$ such that $\left.Q\right|_{a_{1}} \neq \varnothing$ and $\psi^{\mathrm{DS}}\left(\left.b\right|_{a_{1}}\right)\left(\left.Q\right|_{a_{1}}\right) \leq d$. Then, once again by (11) and Lemma 4, there is an action $a_{2} \in A$ such that $\left.Q\right|_{\left(a_{1}, a_{2}\right)} \neq \varnothing$ and $\psi^{\mathrm{DS}}\left(\left.b\right|_{\left(a_{1}, a_{2}\right)}\right)\left(\left.Q\right|_{\left(a_{1}, a_{2}\right)}\right) \leq d$. Repeating this argument leads to a play $p=\left(a_{1}, a_{2}, \ldots\right)$ such that for every $n \in \mathbb{N}$ we have

$$
\left.Q\right|_{\left(a_{1}, \ldots, a_{n}\right)} \neq \varnothing \quad \text { and } \quad \psi^{\mathrm{DS}}\left(\left.b\right|_{\left(a_{1}, \ldots, a_{n}\right)}\right)\left(\left.Q\right|_{\left(a_{1}, \ldots, a_{n}\right)}\right) \leq d .
$$

We argue that $p \in Q$. Since $Q$ is clopen, it is closed. For any $n \in \mathbb{N}$, as $\left.Q\right|_{\left(a_{1}, \ldots, a_{n}\right)} \neq \varnothing$ there is a play $p_{n} \in Q$ such that $p_{n} \succ\left(a_{1}, \ldots, a_{n}\right)$. As the sequence of plays $p_{n}$ converges to $p$, the closedness of $Q$ implies that $p \in Q$, as claimed. 
Since $Q$ is clopen, it is open too. As $p \in Q$, there exists $n \in \mathbb{N}$ such that any play that extends the history $h=\left(a_{1}, \ldots, a_{n}\right)$ belongs to $Q$, i.e. $[h] \subseteq Q$. But then $\psi^{\mathrm{DS}}\left(\left.b\right|_{h}\right)\left(\left.Q\right|_{h}\right)=1$, contradicting (12).

\section{Acknowledgements}

We are grateful to William Sudderth and Andrés Perea for helpful discussions. We would also like to thank the Editor and two anonymous referees for their helpful suggestions. The support for the visit of William Sudderth to Maastricht provided by travel grant nr 040.11.495 of the Netherlands Organisation for Scientific Research (NWO) is acknowledged.

\section{References}

Aryal, G. And StAuber, R. (2014). A note on Kuhn's theorem with ambiguity averse players. Econom. Lett. 125, $110-114$.

Aumann, R. J. (1964). Mixed and behavior strategies in infinite extensive games. In Advances in Game Theory, Annals of Mathematics Studies vol. 52, eds M. Dresher, L. S. Shapley and A. W. Tucker. Princeton University Press, pp. 627-650.

Bingham, N. H. (2010). Finite additivity versus countable additivity. Electron. J. Hist. Probab. Statist. 6, no 1.

CAPRARO, V. AND SCARSINI, M. (2013). Existence of equilibria in countable games: An algebraic approach. Games Econom. Behavior 79, 163-180.

Dubins, L. E. (1974). On Lebesgue-like extensions of finitely additive measures. Ann. Prob. 2, 456-463.

Dubins, L. E. (1975). Finitely additive conditional probabilities, conglomerability and disintegrations. Ann. Prob. 3, 89-99.

Dubins, L. E. And SAVAGE, L. J. (2014). How to Gamble if you Must: Inequalities for Stochastic Processes. New York, Dover Publications. Edited and updated by W. D. Sudderth and D. Gilat.

Dunford, N. And Schwartz, J. T. (1964). Linear Operators, Part I: General Theory. New York, Interscience Publishers.

DE FinetTi, B. (1972). Probability, Induction and Statistics. New York, Wiley.

DE FinetTi, B. (1975). The Theory of Probability. Chichester, J. Wiley and Sons.

Flesch, J., Vermeulen, D. AND Zseleva, A. (2017). Zero-sum games with charges. Games Econom. Behavior 102, 666-686.

Harris, J. H., Stinchcombe, M. B. And Zame, W. R. (2005). Nearly compact and continuous normal form games: Characterizations and equilibrium existence. Games Econom. Behavior 50, 208-224.

KeCHRIS, A. S. (1995). Classical Descriptive Set Theory. Berlin, Springer.

KuHn, H. W. (1953). Extensive games and the problem of information. Ann. Math. Study 28, 193-216.

Loś, J. AND MARCZEWSKI, E. (1949). Extensions of measure. Fundam. Math. 36, 267-276.

Maitra, A. AND Sudderth, W. (1993). Finitely additive and measurable stochastic games. Internat. J. Game Theory 22, 201-223.

Maitra, A. AND SudderTh, W. (1998). Finitely additive stochastic games with Borel measurable payoffs. Internat. J. Game Theory 27, 257-267.

MARINACCI, M. (1997). Finitely additive and epsilon Nash equilibria. Internat. J. Game Theory 26, 315-333.

MAschler, M., Solan, E. And Zamir, S. (2013). Game Theory. Cambridge University Press.

Muraviev, I., Riedel, F. AND SASS, L. (2017). Kuhn's theorem for extensive form Ellsberg games. J. Math. Econom. 68, 26-41.

Purves, R. And Sudderth, W. (1976). Some finitely additive probability. Ann. Prob. 4, 259-276.

RAO, K. P. S. B. And RaO, B. (1983). Theory of Charges: A Study of Finitely Additive Measures. New York, Academic Press.

SaVAGE, L. J. (1972). The Foundations of Statistics. New York, Dover Publications.

Schirokauer, O. And KadAne, J. B. (2007). Uniform distributions on the natural numbers. J. Theoret. Prob. 20, 429-441.

Sudderth, W. (2016). Finitely additive dynamic programming. Math. Operat. Res. 41, 92-108.

TAKAHASHI, M. (1969). A generalization of Kuhn's theorem for an infinite game. J. Sci. Hiroshima Univ. Ser. A-I Math. 33, 237-242. 